\title{
Gerald Shulman digests his award
}

$\mathrm{T}$ he recipient of the 2008 Stanley J. Korsmeyer Award is Gerald I. Shulman, Professor of Medicine and Cellular and Molecular Physiology at Yale University.

This year marks the tenth anniversary of the Stanley J. Korsmeyer Award, presented by the ASCI to one of its members for significant contributions to the understanding of human disease and to mentoring future researchers. Shulman is being recognized for his contributions to furthering our understanding of the mechanisms underlying the pathogenesis of type 2 diabetes mellitus (T2DM). As the recipient of the award, Shulman will receive a $\$ 10,000$ honorarium from the ASCI and will deliver the Korsmeyer Award Lecture at the annual ASCI/AAP Joint Meeting, which is to be held April 25-27, in Chicago, Illinois, USA.

JCI: You are being recognized for your contributions to furthering our understanding of the mechanisms underlying the pathogenesis of T2DM. What do you consider your greatest scientific accomplishment?

Shulman: Our studies of insulin resistance have been built on the work of many other investigators over the last 40 years. I think I am most proud of our development of several novel in vivo magnetic resonance spectroscopy methods that permitted us to elucidate the cellular mechanism for liver and muscle insulin resistance in humans. Using this approach, we were able to disprove a longheld theory regarding fat-induced insulin resistance, involving substrate competition between glucose and fat oxidation, and dis-

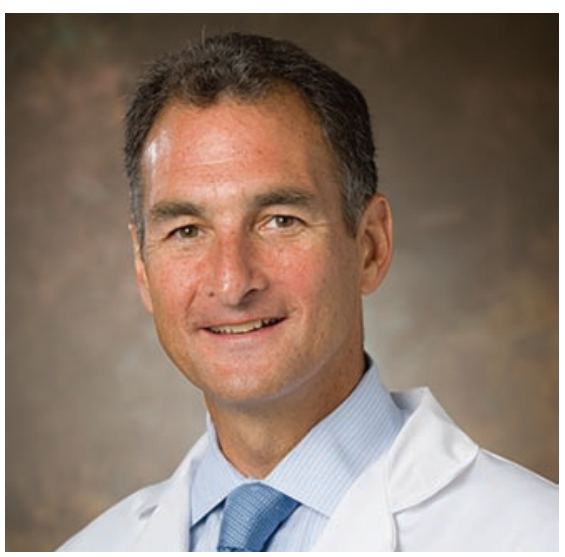

Gerald I. Shulman has been awarded the 2008 Stanley J. Korsmeyer Award for his work on T2DM. covered an entirely new mechanism where net increases in intracellular diacylglycerol, due to increases in fatty acid delivery/synthesis and/or decreased mitochondrial/ peroxisomal fatty acid oxidation, lead to activation of novel PKCs, which in turn inhibits insulin signaling and insulin action in these tissues. We have shown that this unifying hypothesis explains insulin resistance in both obesity and lipodystrophy as well as the insulin-sensitizing effects of thiazolidinediones, exercise, leptin, omega fatty acids, mitochondrial-uncoupling agents, adiponectin, and acetyl CoA carboxylase inhibitors. It has also led to the identification of several novel targets for the treatment and prevention of T2DM.

JCI: When and how did you become interested in metabolic diseases and T2DM in particular?

Shulman: I first became acquainted with diabetes as an eight-year-old attending summer camp in Michigan, which was totally devoted to children with diabetes and where my dad was the camp physician. Watching my fellow campers line up for urine samples and insulin shots every morning, abstaining from candy, and occasionally losing consciousness from hypoglycemia or ketoacidosis left an indelible impression on me regarding the lifelong struggle these individuals have living with this chronic disease. During college, I was first attracted to the inherent beauty of physics and math and majored in biophysics and considered studying this topic in graduate school. However, when I took my first biochemistry and physiology courses in college, which explained all of these pathological processes at the cellular and molecular level, I got hooked on metabolism.

JCI: How do you manage to balance the demands of running a highly successful research laboratory and spending time in the clinic?

Shulman: I think this is a very challenging problem for any physician-scientist who sees patients. My clinical responsibilities include professor rounds on the Yale-New Haven Hospital in-patient medicine service and one month a year attending on the Yale-New Haven Hospital endocrine service. In addition I also have a small clinical practice where I see mostly patients with T1DM and T2DM and unusual cases of severe insulin resistance.

JCI: Do you think there are changes that could be made to improve the transla- tion of basic research into more effective patient care?

Shulman: I think one of the most important components involved in translating basic medical discoveries from the bench to the bedside involves patient-oriented clinical research or the type of clinical research that was traditionally performed in our NIH supported General Clinical Research Centers (GCRCs). The NIH-supported Clinical and Translational Science Awards (CTSAs), which are replacing the GCRCs, were supposed to transform how clinical and translational research is conducted, ultimately enabling researchers to provide new treatments more efficiently and quickly to patients. While I could not agree more with this admirable goal, I think it is ironic that resources such as nursing personnel, support for lab costs, and number of inpatient days for investigators performing patient-oriented research are actually decreasing with this new CTSA program. I think this is a calamity that needs to be corrected immediately before we lose a whole generation of patient-oriented clinical researchers.

JCI: What are your future research plans? Shulman: In addition to our proposed mechanism for insulin resistance, there are several other competing hypotheses for insulin resistance involving alterations in circulating adipocytokines, abdominal obesity, inflammation, and increases in reactive oxygen species. Since each of these other mechanisms have very different therapeutic implications, my lab is currently testing our hypothesis as well as these alternative hypotheses in humans and transgenic/gene knockout mouse models of insulin resistance. I am hopeful that approach will lead to the most effective pharmacologic treatment for T2DM.

JCI: What other contributions to science are you proud of?

Shulman: Training the next generation of physician-scientists. During these past 25 years I have been very fortunate to have worked with a large number of very talented young postdoctoral fellows and students and am very proud that a large number of them have now established their own labs and are now pursuing their own independent research programs.

\section{Karen Honey}

